\title{
COMPACT RIEMANNIAN MANIFOLDS WITH ESSENTIAL GROUPS OF CONFORMORPHISMS
}

\author{
A. J. LEDGER AND MORIO OBATA $\left({ }^{1}\right)$
}

\begin{abstract}
A solution to the following conjecture: A compact connected riemannian $n$-manifold $(n>2)$ with an essential group of conformorphisms is conformorphic to a euclidean $n$-sphere.
\end{abstract}

1. Introduction. Let $(M, g)$ denote a riemannian manifold $M$ together with a riemannian structure $g$ on $M$. In this paper we consider only manifolds (and submanifolds) which are connected and either smooth (i.e. $C^{\infty}$ ) or analytic. The class of a manifold will be mentioned only when the distinction between smooth and analytic is important. Also, unless otherwise stated, we assume $\operatorname{dim} M>2$.

For two riemannian manifolds $(M, g)$ and $\left(M^{\prime}, g^{\prime}\right)$ a diffeomorphism $\theta: M \rightarrow M^{\prime}$ is called a conformorphism if it is conformal, i.e. if there is a function $\rho$ on $M$ such that $\theta^{*} g^{\prime}=e^{2 \rho} g$. If such a $\theta$ exists we say that $(M, g)$ and $\left(M^{\prime}, g^{\prime}\right)$ are conformorphic. If $(M, g)=\left(M^{\prime}, g^{\prime}\right)$, we say $\theta$ is a conformorphism of $(M, g)$. A group $G$ of conformorphisms is called inessential if it is a group of isometries of $(M, \bar{g})$ for some $\bar{g}$ conformal to $g$; otherwise $G$ is essential.

For a smooth (resp. analytic) riemannian manifold $(M, g)$ let $C(M, g)$ (resp. $I(M, g))$ be the group of all conformorphisms (resp. isometries) of $(M, g)$. Then $C(M, g)$ and $I(M, g)$ are Lie transformation groups, with respect to the compactopen topology, which act smoothly (resp. analytically) on $(M, g)$. We write $C_{0}(M, g)$ (resp. $I_{0}(M, g)$ ) for the identity component of $C(M, g)$ (resp. $\left.I(M, g)\right)$.

If $C(M, g)$ is inessential, then it is the group of all isometries of $(M, \bar{g})$ for some $\bar{g}$ conformal to $g$.

The purpose of this paper is to prove the following result:

THEOREM. Let $(M, g)$ be a compact analytic riemannian $n$-manifold $(n>2)$ with finite fundamental group. If the largest connected group $C_{0}(M, g)$ of conformorphisms of $(M, g)$ is essential, then $(M, g)$ is conformorphic to a euclidean $n$-sphere $S^{n}$.

This is a generalization of the following results:

Received by the editors October 15, 1969.

AMS Subject Classifications. Primary 5372, 5325; Secondary 5747.

Key Words and Phrases. Conformorphism, essential (inessential) group of conformorphisms.

(1) The second named author was supported by an NSF Senior Foreign Scientist Fellowship at Lehigh University in 1968-1969.

Copyright (C) 1970, American Mathematical Society 
TheOREM A [1]. Let $(M, g)$ be a compact riemannian $n$-manifold $(n>2)$ with finite fundamental group. If $C_{0}(M, g)$ is essential and transitive, then $(M, g)$ is conformorphic to a euclidean $n$-sphere $S^{n}$.

TheOREM B [7]. Let $(M, g)$ be a conformally flat riemannian $n$-manifold $(n>2)$ with finite fundamental group. If $C_{0}(M, g)$ is essential, then $(M, g)$ is conformorphic to either a euclidean $n$-sphere $S^{n}$ or a once-punctured euclidean $n$-sphere $S^{n}-\left\{p_{\infty}\right\}$ (which is conformorphic to a euclidean $n$-space $E^{n}$ ).

Though our theorem gives a solution to the conjecture, whether or not the conditions 'analytic' and 'finite fundamental group' in our theorem can be relaxed remains an open question.

We now collect some known results for our later use.

TheOREM C [4]. Any compact subgroup of $C(M, g)$ is inessential.

THEOREM D [5]. A separable, metric, locally compact, noncompact, connected group of positive dimension contains a closed subgroup isomorphic to the additive group of all real numbers.

In particular, a noncompact Lie group of positive dimension contains a closed noncompact 1-parameter subgroup.

We write $W$ for the conformal tensor field of type $(1,3)$ on $(M, g)$. Then $W$ is invariant under the action of $C(M, g)$, and if $W \equiv 0$ and $\operatorname{dim} M>3,(M, g)$ is conformally flat. If $\operatorname{dim} M=3, W$ vanishes identically and it is known that there is a tensor field $\tilde{W}$ of type $(0,3)$ constructed from the riemannian structure $g$ such that $(M, g)$ is conformally flat when and only when $\tilde{W}$ vanishes identically. Also $\tilde{W}$ is known to be invariant under the action of $C(M, g)$ in case $\operatorname{dim} M=3$. (More generally, $\tilde{W}$ is constructed for any dimension and is invariant under the action of $C(M, g)$ if $W$ vanishes identically.)

In view of Theorem B to prove our theorem we have only to show, under our assumptions, that $(M, g)$ is conformally flat. In this context we state the following theorem.

THEOREM E [3]. If $W$ is nowhere zero on $(M, g)$ then $C(M, g)$ is inessential.

For $n=3$, Theorem $\mathrm{E}$ holds true if $W$ is replaced by $\tilde{W}$ mentioned above.

2. Local and global conformorphisms. As before, let $(M, g)$ be a smooth or analytic riemannian $n$-manifold. For tensor calculus on $M$ we shall usually follow [2]. For each $p \in M$, let $M_{p}$ (resp. $M_{p}^{*}$ ) denote the tangent (resp. cotangent) space to $M$ at $p$. For $Y \in M_{p}$ and $z \in M_{p}^{*}$ let $Y \wedge z$ be the tensor of type $(1,1)$ defined by $Y \wedge z=Y \otimes z-Z \otimes y$, where $y$ and $Z$ are the images of $Y$ and $z$ respectively, defined by the isomorphism of $M_{p} \rightarrow M_{p}^{*}$ induced by $g$, and $\otimes$ denotes tensor product. 
Let $X$ be a conformal vector field on $M$. Thus $\mathfrak{L}_{X} g=-(2 / n) \delta X g$ where $\delta X=$ - trace $\nabla X$, and $\mathfrak{L}_{X}$ denotes Lie derivation with respect to $X$. Then it is well known (see [6] for example) that for any vector field $Y$ on $M$,

$$
\nabla_{Y} \nabla X=R(Y, X)-\frac{1}{n} d \delta X(Y) I-\frac{1}{n} Y \wedge d \delta X
$$

and

$$
\nabla_{Y} d \delta X=\left(\mathfrak{Q}_{X} L\right) Y .
$$

In (1), $I$ denotes the Kronecker tensor field and in (2)

$$
L=\frac{1}{n-2}\left(\frac{C}{2(n-1)} g-S\right)
$$

where $S$ and $C$ are the Ricci tensor field and scalar curvature respectively.

We remark here that the right-hand side of (2) denotes a 1-form

$$
Z \rightarrow\left(\mathfrak{Q}_{X} L\right)(Y, Z) .
$$

We now consider the following system of differential equations defined along any curve $\gamma$ on $M$,

$$
\begin{gathered}
\nabla_{V} Y=A V, \quad V(\phi)=z(V), \\
\nabla_{V} A=R(V, Y)+z(V) I+V \wedge z, \quad \nabla_{V} z=\left(\left(\nabla_{Y}-A\right) L\right) V
\end{gathered}
$$

where $V$ denotes the tangent field along $\gamma$, and each solution $(Y, A, \phi, z)$ consists of a vector field $Y$, a tensor field $A$ of type $(1,1)$, a function $\phi$, and a cotangent vector field $z$ defined along $\gamma$. We remark that in the last equation of (3), $\nabla_{Y}-A$ is acting as a derivation on $L$. Since the system (3) is linear it follows that there exists a unique solution along $\gamma$ for each set of initial values.

From (1), (2) and (3) we then have

Lemma 1. Let $X$ be a conformal vector field defined on an open set $U$. Then along each curve in $U,(X, \nabla X,-\delta X / n,-d \delta X / n)$ is a solution of (3). If $U$ is connected $X$ is uniquely determined by the values of $X, \nabla X, \delta X$ and $d \delta X$ at any point of $U$.

For any local vector field $X$ at $p \in M$ (i.e. a vector field $X$ defined on some neighbourhood of $p$ ) let $(X)_{p}$ be the germ at $p$ of $X$. Let $c_{p}$ be the set of all germs at $p$ of local conformal vector fields at $p$. If $X$ and $Y$ are local conformal vector fields at $p$ then so are $[X, Y]$ and $a X$ for any real number $a$. It follows that $c_{p}$ has an induced Lie algebra structure. Similarly, there is a Lie algebra structure on the set $c(M, g)$ of all conformal vector fields on $(M, g)$. We now seek a relation between $c_{p}$ and $c(M, g)$. Since only standard methods in the theory of systems of linear differential equations will be used, details will be omitted.

Lemma 2. Suppose $(M, g)$ is analytic. Let $U$ be a normal neighbourhood of a point $p \in M$ and let $(X)_{p} \in c_{p}$. Then $(X)_{p}$ can be extended to a unique conformal 
vector field $Y$ defined on $U$ (i.e. $Y$ is a conformal vector field on $U$ such that $\left.(X)_{p}=(Y)_{p}\right)$.

Proof. With the initial conditions $(Y, A, \phi, z)=(X, \nabla X,-\delta X / n,-d \delta X / n)_{p}$, the system (3) has a unique solution along each geodesic through $p$. Hence we obtain $(Y, A, \phi, z)$ defined on $U$. Moreover, $Y, A, \phi$ and $z$ are analytic. Now from Lemma 1 we have $Y=X$ on some neighbourhood $U_{1}$ of $p$. Hence the tensor field $T=\mathfrak{Q}_{Y} g$ $+(2 / n) \delta Y g$ is zero on $U_{1}$. Since $T$ is analytic on $U$ it follows that $T$ is zero on $U$, and therefore $Y$ is a conformal vector field on $U$ as required. Clearly $(X)_{p}=(Y)_{p}$.

LEMmA 3. Let $(M, g)$ be simply connected and analytic. If $p \in M$ then any $(X)_{p} \in c_{p}$ can be extended to a conformal vector field defined on $M$. This extension defines an isomorphism of $c_{p}$ onto $c(M, g)$.

Proof. Let $\gamma: J \rightarrow M$ be a curve on $M$ with initial point $p$, and let $(Y, A, \phi, z)$ be the solution of (3) along $\gamma$ with initial conditions

$$
(Y, A, \phi, z)_{p}=(X, \nabla X,-\delta X / n,-d \delta X / n)_{p} .
$$

Then it follows from Lemma 1 and Lemma 2 that for each $t \in J$ there exists a unique local conformal vector field $Y_{t}^{\prime}$ defined on some neighbourhood of $\gamma(t)$ such that

$$
\left(Y_{t}^{\prime}, \nabla Y_{t}^{\prime},-\delta Y_{t}^{\prime} / n,-d \delta Y_{t}^{\prime} / n\right)_{\gamma(t)}=(Y, A, \phi, z)_{\gamma(t)} .
$$

Then, by standard deformation theory, it results that the value of $Y_{\gamma(1)}^{\prime}$ at $\gamma(1)$ depends only on the homotopy class of $\gamma$. Since $M$ is assumed to be simply connected we obtain in this way a vector field $Y^{\prime}$ on $(M, g)$ which is clearly conformal and is an extension of $(X)_{p}$. The existence and uniqueness of each extension implies that $c_{p}$ and $c(M, g)$ are isomorphic.

In the remainder of this section our main purpose is to obtain sufficient conditions for $C_{0}(M, g)$ and $C_{0}\left(M^{\prime}, g^{\prime}\right)$ to be isomorphic, where $M^{\prime}$ is an open submanifold of $M$ with induced riemannian structure $g^{\prime}$. The method depends essentially, on proving that the corresponding Lie algebras are isomorphic by means of Lemma 3.

Let $G$ be a Lie group acting as an effective transformation group on a smooth (resp. analytic) manifold $M$. We write $\alpha_{G}$ for the monomorphism of $L G$ (the Lie algebra of $G$ ) into the Lie algebra of all smooth (resp. analytic) vector fields on $M$.

LemMA 4. Let $G$ and $G_{1}$ be two connected Lie groups acting as effective transformation groups on a manifold $M$. Suppose $G_{1}$ (as an abstract group) is a subgroup of $G$ and suppose $\alpha_{G_{1}} L G_{1}=\alpha_{G} L G$. Then the inclusion map $G_{1} \rightarrow G$ is an analytic isomorphism.

Proof. Since $\alpha_{G}$ and $\alpha_{G_{1}}$ are monomorphisms with the common image $\alpha_{G} L G$ there is a unique isomorphism $\beta: L G_{1} \rightarrow L G$ such that $\alpha_{G_{1}}=\alpha_{G} \circ \beta$. Then for all $t \in \boldsymbol{R}, X \in L G_{1}$, and $p \in M$ we have $(\exp t X)(p)=(\exp t \beta(X))(p)$, where the expo- 
nential maps are defined with respect to the corresponding Lie groups. Hence, since $G$ is effective, for sufficiently small $t$, $\exp t X=\exp t \beta(X)=B(\exp t X)$, where $B$ is the local isomorphism of groups induced by $\beta$. Thus the inclusion map of $G_{1} \rightarrow G$ is clearly analytic at the identity of $G_{1}$ and hence everywhere. Since $G_{1}$ is connected and $L G_{1}=L G$ the lemma follows.

Lemma 5. Let $(M, g)$ be simply connected and analytic. Let $\left(M^{\prime}, g^{\prime}\right)$ be an open submanifold of $(M, g)$ with induced riemannian structure $g^{\prime}$, and suppose $M^{\prime}$ is invariant under the action of $C_{0}(M, g)$. Then the map $\sigma: c(M, g) \rightarrow c\left(M^{\prime}, g^{\prime}\right)$, defined by restricting all conformal vector fields on $(M, g)$ to $\left(M^{\prime}, g^{\prime}\right)$, is an isomorphism.

Proof. The map $\sigma$ is clearly linear. Also, by Lemma 3, $\sigma$ is surjective. Finally, $\sigma$ is injective since, by Lemma 3 , each element in $c\left(M^{\prime}, g^{\prime}\right)$ extends uniquely to an element in $c(M, g)$. Thus $\sigma$ is an isomorphism.

LEMMA 6. With the notation and assumptions of Lemma 5, suppose $M$ is compact. Then the map $r: C_{0}(M, g) \rightarrow C_{0}\left(M^{\prime}, g^{\prime}\right)$ defined by restricting to $\left(M^{\prime}, g^{\prime}\right)$ all conformorphisms in $C_{0}(M, g)$ is an analytic isomorphism.

Proof. Since by restriction $C_{0}(M, g)$ acts conformally on $\left(M^{\prime}, g^{\prime}\right)$ it follows that if $X \in \alpha_{C(M, g)} L C(M, g)$ then $\sigma(X) \in \alpha_{C\left(M^{\prime}, g^{\prime}\right)} L C\left(M^{\prime}, g^{\prime}\right)$. Hence

$$
\sigma\left(\alpha_{C(M, g)} L C(M, g)\right) \subset \alpha_{C\left(M^{\prime}, g^{\prime}\right)} L C\left(M^{\prime}, g^{\prime}\right) .
$$

Again, since $M$ is compact $\alpha_{C(M, g)}$ is an isomorphism of $L C(M, g)$ onto $c(M, g)$. Thus if $X \in \alpha_{C\left(M^{\prime}, g^{\prime}\right)}\left(L C\left(M^{\prime}, g^{\prime}\right)\right)$ then $\sigma^{-1}(X) \in \alpha_{C(M, g)}(L C(M, g))$. Hence

$$
\sigma\left(\alpha_{C(M, g)} L C(M, g)\right)=\alpha_{C\left(M^{\prime}, g^{\prime}\right)} L C\left(M^{\prime}, g^{\prime}\right) .
$$

Now the map $r$ is clearly a homomorphism. Moreover, since $M$ is analytic and $M^{\prime}$ is open, $r$ is injective. Let $G=r\left(C_{0}(M, g)\right)$ and let $G$ have the Lie group structure such that $r$ is analytic. Then $G$ acts as an effective Lie transformation group on $M^{\prime}$ and from (4) $\alpha_{G}(L G)=\alpha_{C\left(M^{\prime}, g^{\prime}\right)} L G\left(M^{\prime}, g^{\prime}\right)$. Hence by Lemma 4, the inclusion map $G \rightarrow C_{0}\left(M^{\prime}, g^{\prime}\right)$ is an analytic isomorphism. The lemma follows immediately.

We finally prove a result on isometries.

LemMA 7. A closed subgroup $H$ of $I(M, g)$ is compact if and only if there exists a compact orbit $H(p)$ in $M$.

Proof. If $H$ is compact then clearly every orbit is compact. Conversely, suppose $H$ is closed and $H(p)$ is compact. Let $K$ be the isotropy subgroup of $H$ at $p$. Then $K$ is closed in $H$ and hence in $I(M, g)$. But $K$ is contained in the isotropy subgroup of $I(M, g)$ at $p$ which is compact. Hence $K$ is compact. Now $H(p)$ is closed in $M$ and hence is a locally compact, Hausdorff subspace of $M$. Then $H(p)$ is homeomorphic to $H / K$ by [1, Chapter II, Theorem 3.2]. It follows immediately that $H$ is compact. 
3. Proof of theorem. Let $(M, g)$ be a manifold which is not conformally flat and let $M_{1}=\left\{p \in M: W_{p} \neq 0\right\}$. In case $\operatorname{dim} M=3, M_{1}=\left\{p \in M: \tilde{W}_{p} \neq 0\right\}$. Then $M_{1}$ is an open subset of $M$ and any component $M^{\prime}$ of $M_{1}$ is an open submanifold of $M$. Also, since $W$ and $\tilde{W}$, when $W \equiv 0$, are invariant under the action of $C(M, g)$ it follows that $M^{\prime}$ is fixed under this action. Hence $M^{\prime}$ is fixed under the action of $C_{0}(M, g)$.

Suppose $(M, g)$ is also compact, simply connected and analytic. Let $g^{\prime}$ be the restriction of $g$ to $M^{\prime}$. Then, by the map $r$ of Lemma 7 we may identify $C_{0}(M, g)$ with $C_{0}\left(M^{\prime}, g^{\prime}\right)$, and hence, by Theorem E, $C_{0}(M, g)=I_{0}\left(M^{\prime}, \bar{g}^{\prime}\right)$, for some $\bar{g}^{\prime}$ conformal to $g^{\prime}$. We wish to prove that $C_{0}(M, g)$ is compact, and this will be done by a contradiction argument. Thus, suppose $C_{0}(M, g)$ is noncompact. Then by Theorem $\mathrm{D}, C_{0}(M, g)$ contains a closed noncompact 1-parameter subgroup $H$. Then $H$ is closed in $I_{0}\left(M^{\prime}, \bar{g}^{\prime}\right)$ and hence, for $p \in M^{\prime}$, the orbit $H(p)$ is a closed submanifold of $M^{\prime}$. Since $H$ is closed and noncompact, by Lemma 7, $H(p)$ is noncompact and is homeomorphic to $H$ itself by the natural projection $H \rightarrow H(p)$.

Let $X$ be the conformal vector field on $(M, g)$ corresponding to the 1-parameter group $H$. Then $X$ is nowhere zero in $M^{\prime}$, since $H(p)$ is homeomorphic to $H$.

Now on $M$ we put

$$
\left.F(p)=|X \otimes X \otimes W|_{p}^{2} \quad \text { (resp. } \tilde{F}(p)=|X \otimes X \otimes X \otimes \tilde{W}|_{p}^{2}\right),
$$

where we write $|T|$ for the magnitude of any tensor $T$ with respect to $g$.

Since $X$ and $W$ are invariant by $H$, so is $X \otimes X \otimes W$. As it is of type (3.3), its magnitude is invariant by $H$ as well. Thus we have

$$
\mathfrak{L}_{X} F=0 \quad\left(\text { resp. } \mathfrak{Q}_{X} \tilde{F}=0\right. \text { ). }
$$

Hence $F$ is a nonzero constant on $H(p), p \in M^{\prime}$. Suppose $q \in \mathrm{Cl} H(p), p \in M^{\prime}$, where $\mathrm{Cl} H(p)$ denotes the closure of $H(p)$ in $M$.

Then $F$ is a nonzero constant on $\mathrm{Cl} H(p)$ and hence $F(q) \neq 0$. Thus $q \in M^{\prime}$. Consequently, since $H(p)$ is closed in $M^{\prime}$, we have $q \in H(p)$. Thus $H(p)$ is a closed orbit in the compact space $M$ and hence is a compact submanifold of $M$. It follows from Lemma 7 that $H$ is compact and so we have a contradiction. Thus $C_{0}(M, g)$ must be compact and, therefore, inessential by Theorem C. It follows that if $(M, g)$ is compact, simply connected, and analytic with essential $C_{0}(M, g)$, then it is conformally flat.

The extension to manifolds with finite fundamental group is an easy consequence of Theorem $B$.

\section{REFERENCES}

1. B. Ba, Structures presque complexes, structures conformes et dérivations, Topologie et Géométrie Différentielle, vol. 8, Centre Nationale Recherche Sci., Paris, 1966. MR 37 \#5809.

2. S. Helgason, Differential geometry and symmetric spaces, Pure and Appl. Math., vol. 12, Academic Press, New York, 1962. MR 26 \#2986.

3. V. Hlavatý, Zur Konformgeometrie, Proc. Acad. Amsterdam 38 (1935), 281-286. 
4. S. Ishihara, Groups of projective transformations and groups of conformal transformations, J. Math. Soc. Japan 9 (1957), 195-227. MR 20 \#311.

5. D. Montgomery and L. Zippin, Existence of subgroups isomorphic to the real numbers, Ann. of Math. (2) 53 (1951), 298-326. MR 13, 911.

6. T. Nagano, On conformal transformations of Riemannian spaces, J. Math. Soc. Japan 10 (1958), 79-93. MR 22 \#1859.

7. M. Obata, Conformally flat Riemannian manifolds admitting a one-parameter group of conformal transformations, J. Differential Geometry 4 (1970) (to appear).

THE UNIVERSITY OF LIVERPOOL, LIVERPOOL, ENGLAND

LEHIGH UNIVERSITY, Bethelhem, Pennsylvania 18015

TOKYO METROPOLITAN UNIVERSITY, TOKYO, JAPAN 